\title{
Robust control of the distributed solar collector field ACUREX using MPC for tracking. ${ }^{\star}$
}

\author{
D. Limon* I. Alvarado* T. Alamo* M. Ruiz* \\ E. F. Camacho* \\ * Dpto. de Ingeniería de Sistemas y Automática, Universidad de \\ Sevilla, Avda de los Descubrimeintos s/n 41092, Sevilla (limon, \\ alvarado, alamo, arahal, camacho@cartuja.us.es).
}

\begin{abstract}
This paper presents the application of a robust model predictive control for tracking of piece-wise constant references (RMPCT) to a distributed collector field, ACUREX, at the solar power plant of PSA (Solar Plant of Almería). The main characteristic of a solar power plant is that the primary energy source, solar radiation, cannot be manipulated. Solar radiation varies throughout the day, causing changes in plant dynamics and strong disturbances in the process. The real plant is assumed to be modeled as a linear system with additive bounded uncertainties on the states. Under mild assumptions, the proposed RMPCT can steer the uncertain system in an admissible evolution to any admissible steady state, that is, under any change of the set point. This allows us to reject constant disturbances compensating the effect of then changing the setpoint.
\end{abstract}

\section{INTRODUCTION}

ACUREX is a solar plant located at Tabernass desert (Spain) and it is one of the available experimental plants of the PSA complex (Solar plant of Almería). It consists of a series of parabolic mirrors that reflect solar radiation onto a pipe where oil gets heated while circulating. The objective of the control system in a distributed collector field is to maintain the outlet oil temperature at a desired level despite of disturbances such as changes in the solar irradiance level (caused by clouds), mirror reflectivity or inlet oil temperature. Since solar radiation cannot be adjusted, this can only be achieved by adjusting the oil flow.

In this paper, the control of the ACUREX plant has been carried out by means of a RMPCT recently proposed in (Limon et al. [2007]), the feasibility, stability and asymptotical convergence of the proposed controller for any admissible setpoint is achieved by adding an artificial steady state as decision variable, penalizing the deviation between this artificial steady state and the real one in the cost function and using as a terminal constraint an invariant set for tracking. Robust constraint satisfaction is guaranteed by tube-based approach; a nominal control problem is defined whose solution (a trajectory) defines the center of a tube; and where the 'cross-section' of the tube is an invariant set. The constraints for the nominal system would be those that the tube satisfies the real constraints. The state trajectory of the controlled system will be forced, by the control to lie in this tube. At each time, a new tube is determined by solving a control optimization in which, the decision variables are the initial state of the nominal

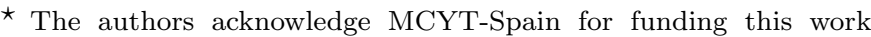
DPI2007-66718-C04-01. system, the control sequence over a finite horizon and the artificial steady state.

The paper is organized as follows. First, in section 2 a brief description of the distributed solar collector field is given. The section 3 is dedicated to presenting the RMPCT. After that, section 4 presents the identification procedure. Section 5 shows the results obtained in the plant using he RMPCT. Section 6 shows results obtained in the plant using the RMPCT plus the cancellation offset loop, and the conclusions are presented in Section 7.

Notation: A definite positive matrix $T$ is denoted as $T>0$ and $T>P$ denotes that $T-P>0$. For a given symmetric matrix $P>0,\|x\|_{P}$ denotes the weighted Euclidean norm of $x$, i.e. $\|x\|_{P}=\sqrt{x^{T} P x} .(a, b) \triangleq\left[a^{\prime}, b^{\prime}\right]^{\prime}$. Consider $a \in \mathbb{R}^{n_{a}}, b \in \mathbb{R}^{n_{b}}$, and set $\Gamma \subset \mathbb{R}^{n_{a}+n_{b}}$, then projection operation is defined as $\operatorname{Proj}_{a}(\Gamma)=\left\{a \in \mathbb{R}^{n_{a}}: \exists b \in \mathbb{R}^{n_{b}},(a, b) \in \Gamma\right\}$. Given two sets $\mathcal{U}$ and $\mathcal{V}$, such that $\mathcal{U} \subset \mathbb{R}^{n}$ and $\mathcal{V} \subset \mathbb{R}^{n}$, the Minkowski sum is defined by $\mathcal{U} \oplus \mathcal{V} \triangleq\{u+v \mid u \in \mathcal{U}, v \in \mathcal{V}\}$, the Pontryagin set difference is: $\mathcal{U} \ominus \mathcal{V} \triangleq\{x \mid x \oplus \mathcal{V} \subseteq \mathcal{U}\}$. for a given $\lambda, \lambda \mathcal{X}=\{\lambda x: x \in \mathcal{X}\}$. Let $\mathbf{t}$ be a generic vector defined as $\mathbf{t} \triangleq\{t(0), t(1), \ldots\}$.

\section{DESCRIPTION OF THE SOLAR POWER PLANT ACUREX}

The distributed collector field involves the collection of solar energy and its transfer to a fluid piped through the system. The energy collected is transferred to a storage tank, which can be tapped when conditions demand, on to either a steam generator for electrical power generation or the heat exchanger of a desalination plant. It mainly consists of a pipeline through which oil is flowing and onto which the sun's rays are concentrated by means of parabolic mirrors, which follow the sun by rotating on one axis, in order to heat the oil. It is composed by 480 modules 
arranged in 20 lines which form 10 parallel loops. Figure 2 shows a diagram of the solar collector field. The field is also provided with a sun-tracking mechanism which causes the mirrors to revolve around an axis parallel to that of the pipeline. Each of the loops mentioned above is formed by four 12-module collectors, suitably connected in series. The loop is $172 \mathrm{~m}$ long, the active part of the loop (exposed to concentrated radiation) measuring $142 \mathrm{~m}$ and the passive part $30 \mathrm{~m}$. A fundamental feature of a solar power plant is

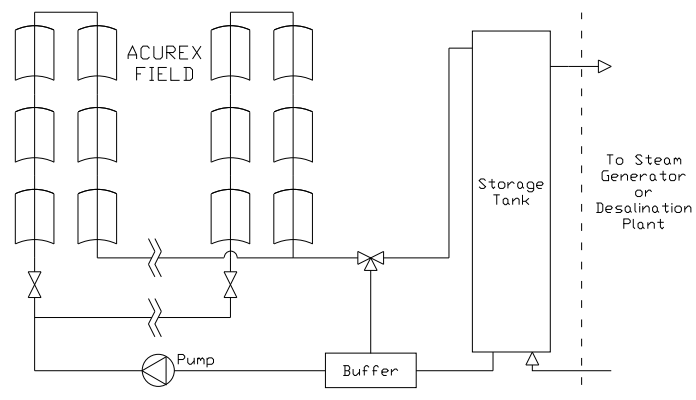

Fig. 1. Acurex scheme.

that the primary energy source, while it is variable, cannot be manipulated. The intensity of solar radiation from the sun, in addition to its seasonal and daily cyclic variations, is also dependent on atmospheric conditions such as cloud cover, humidity and air transparency. In order to obtain a suitable experimental data for the identification, the experiments must be executed in a period where the environmental variables were constant, i. e. the changes in the output are only produced by the control input. The dynamical characterization of the field has been done in both the time and frequency domains. Open-loop step responses have been obtained at several operating points. One of these responses is shown in Figure 2 (a change in oil flow from 8 to $7 \frac{l}{s}$ was performed). As can be seen, the step response shows that the behavior of the field can be modeled by a low-order model as shown in previous works done in the plant (F. et al. [1994a]), although the plant dynamics are more complex (presence of antiresonance modes) as can be seen in the frequency response plot shown in the figure 2 .

The use of high-order models with standard parameter estimation algorithms often produces wrong results because of the divergence of the estimated parameters (F. et al. [1994b]). Using low-order models with Model Predictive control algorithms, acceptable behavior can be obtained.

\subsection{Series feedforward compensation}

The models relate changes in outlet temperature to changes in oil flow. The outlet temperature of the plant,
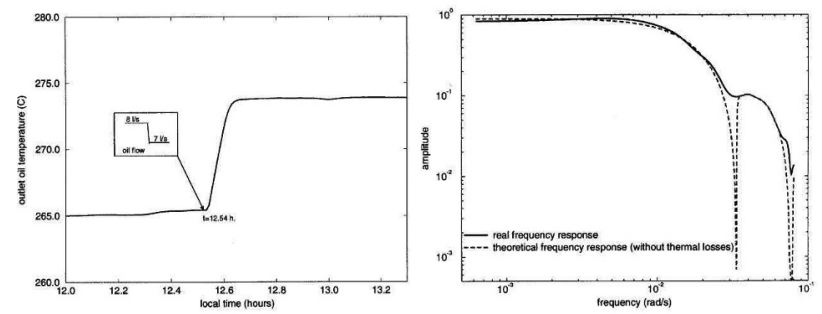

Fig. 2. Dynamical characterization of the field. however, is also influenced by changes in system variables such as solar radiation and fluid inlet temperature.

In order to account for these disturbances, a series feedforward controller has been introduced. The feedforward is calculated from steady state relationships, which make an adjustment in the fluid flow input, aimed to eliminate the change in outlet temperature caused by the variations in solar radiation and inlet temperature. The calculation employed is:

$$
\text { flow }=\frac{a_{1} I r r-a_{2}\left(F F_{r e f}-a_{3}\right)-a_{4}}{F F_{\text {ref }}-T_{\text {in }}}
$$

where $a_{1}, a_{2}, a_{3}, a_{4}$ are parameters, flow is the oil flow, $F F_{\text {ref }}$ is the temperature setpoint, $T_{i n}$ is the inlet oil temperature and $\mathrm{Irr}$ is the effective solar radiation. The feedforward is placed in series with the robust controller which provides the signal $F F_{\text {ref }}$ (see figure 5).

Although exact elimination obviously cannot be achieved, this term helps to preserve the validity of the assumed system models and provides control benefits when disturbances in solar radiation and fluid inlet temperature occur. The feedforward is considered as a part of the models and thus the input signal for the plant model is the setpoint temperature for the feedforward term and not the oil flow (this is calculated by the feedforward controller). The feedforward term is explained extensively in (Berenguel et al. [1993], F. et al. [1994a,b]).

\section{RMPCT}

\subsection{Problem description}

Consider the following uncertain discrete-time linear timeinvariant system:

$$
x^{+}=A x+B u+w \quad y=C x+D u
$$

Where $x \in \mathcal{X} \subseteq \mathbb{R}^{n}, u \in \mathcal{U} \subseteq \mathbb{R}^{m}$ and $y \in \mathcal{Y} \subseteq \mathbb{R}^{p}$ is the current state, the current input and the current output of the system; $x^{+}$is the successor state $; w \in \mathcal{W} \subseteq \mathbb{R}^{n}$ is an unknown and bounded state disturbance. $x(\bar{k}), u(k)$, $w(k)$ denote the state, the input and the disturbance at sampling time $k$ of the system (1). $\mathcal{X}, \mathcal{U}, \mathcal{Y}, \mathcal{W}$ are convex polyhedra containing the origin in its interior.

Assumption 1. System (1) should verify:

- $(A, B, C, D)$ are known and $(A, B)$ controllable.

- The state is accessible.

The proposed controller will be based on the response of the nominal system obtained from (1) by neglecting the disturbances $w$.

$$
\bar{x}^{+}=A \bar{x}+B \bar{u} \quad \bar{y}=C \bar{x}+D \bar{u}
$$

$\bar{x}(i)=\bar{\phi}(i ; \bar{x}, \overline{\mathbf{u}})$ represent the evolution of the nominal state at time $i$ if its initial nominal state is $\bar{x}$ and the control sequence is $\mathbf{u}$

To counteract the disturbances it is desirable to force the trajectory to lie close to the nominal trajectory; this can be done by choosing the control u to satisfy:

$$
u=\bar{u}+K e \quad e \triangleq(x-\bar{x})
$$


where $e$ denotes the control error between the state and the state of the nominal system.

The error $e$ satisfies the difference equation:

$$
e^{+}=A_{K} e+w ; \quad A_{K}=(A+K C)
$$

Assumption 2. The feedback control matrix $K$ is such that $A_{K}$ is stable (Hurwitz).

Because $A_{K}$ is Hurwitz, there exists a robust positively invariant set $\mathcal{Z}$ (Kolmanovsky and Gilbert [1998], Rakovic et al. [2005]) for the system (4) that satisfies

$$
A_{K} \mathcal{Z} \oplus \mathcal{W} \subseteq \mathcal{Z}
$$

There are several methods for determining the robust positively invariant set as the ones introduced in (Blanchini [1999], Rakovic et al. [2005], Rakovic [2007]).

Since $e(0) \in \mathcal{Z}$ implies $e(i) \in \mathcal{Z} \quad \forall i \in \mathbb{N}$, an obvious consequence is:

Proposition 1. If the initial real and nominal system states, satisfy $e(0)=x(0)-\bar{x}(0) \in \mathcal{Z}$, then $x(i) \in \bar{x}(i) \oplus$ $\mathcal{Z} \forall i \in \mathbb{N}$, all admissible disturbance sequences $\mathbf{w}$.

Theorem 1. (Mayne et al. [2005]). Suppose the initial system and nominal system states all lie in $\mathcal{X}$ and satisfy $e(0)=x(0)-\bar{x}(0) \in \mathcal{Z}$. In addition, if the initial state $\bar{x}(0)$ and control sequence $\overline{\mathbf{u}}$ of the nominal system satisfy the tighter constraints $\bar{x}(i)=\bar{\phi}(i ; x(0), \overline{\mathbf{u}}) \in \mathcal{X} \ominus \mathcal{Z}$ and $\bar{u}(i) \in \mathcal{U} \ominus K \mathcal{Z}$ for all $i \in \mathbb{N}$. The state $x(i)$ and the control $u(i)=\bar{u}(i)+K(x(i)-\bar{x}(i))$ of the real system $(1)$ satisfy the original constraints $x(i) \in \mathcal{X}$ and $u(i) \in \mathcal{U}$ for all $i \in \mathbb{N}$ and all admissible disturbance sequences $\mathbf{w}$.

\subsection{Controller formulation}

In order to ensures the feasibility of the problem when the set point in changed, an artificial reachable steady state is introduced $\left(\bar{x}_{s}, \bar{u}_{s}\right)$ as a decision variable in our optimization problem. Then if the new setpoint point is not reachable the system will be leaded to a different reachable setpoint (the artificial steady state). It is clear that doing this the feasibility is ensured but the convergence can be lost. To avoid this, a term $\left\|\bar{x}_{s}-x_{s}\right\|_{T}^{2}$ (the offset cost) that penalizes the deviation between the desired steady state and the artificial one is added in the cost function:

$$
\begin{aligned}
V_{N}\left(x, x_{s} ; \overline{\mathbf{u}}, \bar{x}, \theta\right) & =\sum_{k=0}^{N-1}\left\|\bar{x}(i)-\bar{x}_{s}\right\|_{Q}^{2}+\left\|\bar{u}(i)-\bar{u}_{s}\right\|_{R}^{2} \\
& +\left\|\bar{x}(N)-\bar{x}_{s}\right\|_{P}^{2}+\left\|\bar{x}_{s}-x_{s}\right\|_{T}^{2}
\end{aligned}
$$

This cost penalizes the deviation between the predicted trajectory and the artificial steady state along the horizon $N$ and the deviation between the artificial and the desired steady state $x_{s} . \theta$ is a variable that determine the artificial steady state $\left(\bar{x}_{s}, \bar{u}_{s}\right)=M \theta$ (Limon et al. [2005b]).

The formulation of the optimal problem $\mathcal{P}_{N}\left(x, x_{s}\right)$ is:

$$
\begin{aligned}
\min _{\overline{\mathbf{u}}, \bar{x}, \theta} & V_{N}\left(x, x_{s} ; \overline{\mathbf{u}}, \bar{x}, \theta\right) \\
\text { s.t. } & \bar{x} \in x \oplus(-\mathcal{Z}) \\
& \bar{x}(i) \in \overline{\mathcal{X}}=\mathcal{X} \ominus \mathcal{Z} \\
& \bar{u}(i) \in \overline{\mathcal{U}}=\mathcal{U} \ominus K \mathcal{Z} \\
& (\bar{x}(N), \theta) \in \Omega_{\bar{K}}^{w}
\end{aligned}
$$

where $\Omega_{\bar{K}}^{w}$ is an invariant set for tracking associated to the linear control law described in (Limon et al. [2005b]) with a gain $\bar{K}$ that can be different to $K$.

Due to the constraints set does not depend on $x_{s}$, the optimization problem $\mathcal{P}_{N}\left(x, x_{s}\right)$ has a feasible solution $\forall x \in \mathcal{X}_{N} \subset \mathbb{R}^{n}$. The size of that set depends, among other things, on the size of $\Omega_{\bar{K}}=\operatorname{Proj}_{x}\left(\Omega_{\bar{K}}^{w}\right)$. As bigger is this set, bigger is $\mathcal{X}_{N}$ (Limon et al. [2005a]). Because of that $\forall x \in \mathcal{X}_{N}$, The optimization problem has a solution denoted by *, so, $V_{N}^{*}\left(x, x_{s}\right)$ would be the optimal cost, $\overline{\mathbf{u}}^{*}\left(x, x_{s}\right), \bar{x}^{*}\left(x, x_{s}\right)$ and $\theta^{*}\left(x, x_{s}\right)$ would be the optimal value of the decision variables, $\overline{\mathbf{x}}^{*}\left(x, x_{s}\right)$ would be the nominal optimal trajectory and $\left(\bar{x}_{s}^{*}\left(x, x_{s}\right), \bar{u}_{s}^{*}\left(x, x_{s}\right)\right)$ would be the optimal artificial reference.

The control action is calculated from the optimal solution as follows

$$
\kappa_{N}\left(x, x_{s}\right)=K\left(x-\bar{x}^{*}\left(x, x_{s}\right)\right)+\bar{u}^{*}\left(0 ; x, x_{s}\right)
$$

where $\bar{u}^{*}\left(0 ; x, x_{s}\right)$ is the first component of $\overline{\mathbf{u}}^{*}\left(x, x_{s}\right)$.

Remark 2. (controller properties). This controller ensures the stability, the convergence and the constraints satisfaction for any change of the setpoint at any sample time. Moreover, if the provided setpoint is not admissible the system will be leaded to the closest equilibrium state in the sense that the offset cost is minimized (Alvarado [2007])

It is important that, in the case that the disturbances set, the constraints set, the robust invariant set $\mathcal{Z}$ and the invariant set $\Omega_{\bar{K}}^{w}$ were polyhedra, the proposed problem can be formulated as a QP (Quadratic Programming Problem), that can be easily solved using efficient algorithms (Camacho and Bordons [2004], Luenberger [1989]).

More information about the controller and how the set can be calculated can be found in (Limon et al. [2005a, 2007], Alvarado [2007])

\section{IDENTIFYING THE PLANT PLUS THE FEEDFORWARD}

Due to the chosen controller is a RMPCT, a space state representation with additive disturbances of the plant (1) is needed.

In this case $u$ is $F F_{r e f}, w$ is the model error and $y$ is the output temperature $T_{\text {out }}$.

To identify the plant, as it was aforementioned, a period where the environmental variables were constant is chosen, thus, the changes in the output are going to be only produced by the changes in the input. (see an example in figure 3). A few low order models with different delays have been identified by the least square method and finally the model with the smaller identification error was a first order model without delay:

$$
\begin{array}{ll}
A=0.8656 & B=0.1251 \\
C=1 & D=0
\end{array}
$$

The set of possible values of $w$ has been calculated computing the error between the real data and the modeled one from some experiments (see figure 4). The obtained set is $|w| \leq 5$ The constraints set for that system are:

$$
\begin{aligned}
& 100 \leq u \leq 350 \\
& 0 \leq y \leq 300
\end{aligned}
$$



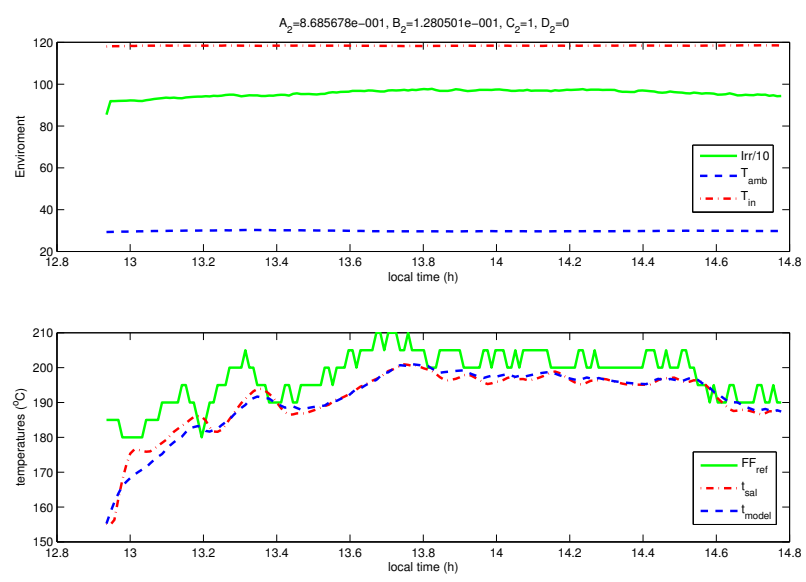

Fig. 3. Plant identification.
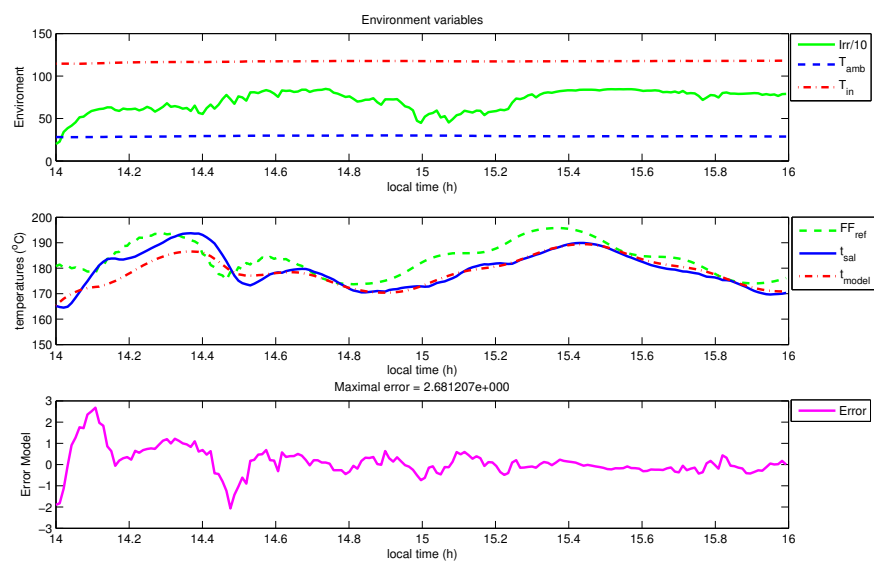

Fig. 4. Maximal error $w_{\max }$.

The supervision module of the loop adds a couple of constraints:

- The maximal difference between the temperatures of each collector is $80^{\circ}$,

$$
T_{\text {out }}-T_{\text {in }} \leq 80
$$

- The constraint set for the flow is

$$
2 \leq \text { flow } \leq 10 \frac{l}{s}
$$

These constraints limits the reachable set of output temperatures of the plant.

\section{APPLYING THE ROBUST MPC FOR TRACKING}

The proposed RMPCT is used to manipulate $F F_{r e f}$ ensuring robust admissibility, stability, disturbance rejection and setpoint tracking. Figure 5 shows the general scheme of the controlled plant.

The RMPCT has been adjusted, via simulation, to con-

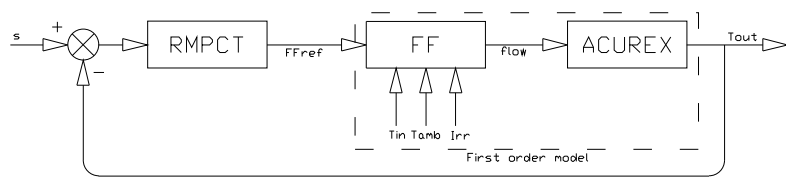

Fig. 5. RMPCT scheme.

trol the plant in order to be as fast as posible without overshooting. The provided nonlinear model is based on partial differential equations (Berenguel et al. [1993]).

The controller parameters are:

$$
\begin{aligned}
& M P C \# 1 \\
& \mathrm{Q}_{\mathrm{z}}=1, \mathrm{R}_{\mathrm{z}}=1, \mathrm{~K}=-0.36726 \\
& \mathcal{Z}=(-28.0174,28.0174) \\
& \mathrm{Q}=1000, \mathrm{R}=1, \overline{\mathrm{K}}=-6.40848 \\
& \overline{\mathcal{Y}}_{e q} \in(107.34,271.71) \\
& \overline{\mathcal{U}}=\mathrm{U} \ominus \mathrm{KZ}=(110.29,339.71) \\
& X_{n}=(0.00,300.00)
\end{aligned}
$$

- The local controller gain to determined $\mathcal{Z}$ is the one corresponding to the LQR with $Q=Q_{z}$ and $R=R_{z}$. It is not the one who makes $\mathcal{Z}$ minimal in order not to produce overshooting.

- $Q$ and $R$ are the matrices weights of the cost function, they are also used to calculate the controller gain, using the LQR, to calculate the invariant set for tracking used as the terminal constraint.

- $\overline{\mathcal{Y}}_{e q}$ is the set of the admissible setpoints.

- $\overline{\mathcal{U}}$ is the set of the admissible control actions for the nominal system.

- $X_{n}$ is the domain of attraction.

In the following figures, in the upper graphic is shown: the reference (solid line), the artificial reference (dasheddot line) and the output (dashed line) and in the lower one, the real disturbances (the radiation, the corrected radiation (both in dashed line), the temperature of the input $T_{i n}$ (dashed-dot line)) and the an estimation of the disturbance $v_{\text {est }}$ (solid line).

Figure 6 shows the evolution of the plant controlled by the $M P C \# 1$ under good conditions.
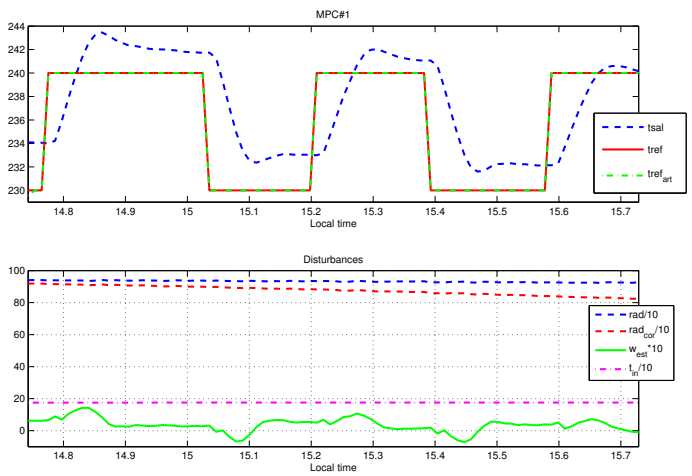

Fig. 6. $M P C \# 1$ without disturbances.

In the following subsection the behavior of the controlled plant under different situations are show.

\subsection{Radiation disturbance}

Figure 7 shows the evolution of the plant in presence of clouds.

The parameters of the controller are the same as in the previous case

The maximal possible error between the real state and the reference in permanent regime is the $28^{0}$ (the size of $\mathcal{Z}$ ) 

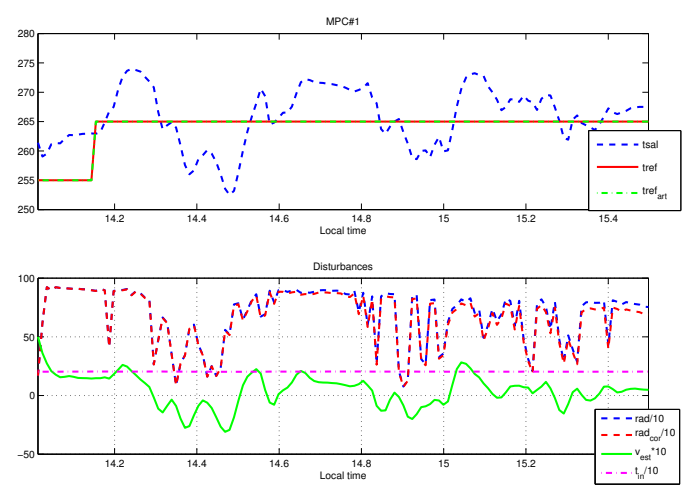

Fig. 7. $M P C \# 1$ with radiation disturbance.

that can be a non admissible error, to avoid this, there are two possible solutions.

- Try to do the local controller $K$ more aggressive, with the risk of overshooting.

- Look for a method that, changing the setpoint, cancel the error. (See section 6)

\subsection{Pyrometer sensor error}

In this case the controlled used is more aggressive, the parameters are:

$$
\begin{aligned}
& M P C \# 2 \\
& \mathrm{Q}_{\mathrm{z}}=10, \mathrm{R}_{\mathrm{z}}=1, \mathrm{~K}=-1.81804 \\
& Z=(-13.7275,13.7275) \\
& \mathrm{Q}=1000, \mathrm{R}=1, \overline{\mathrm{K}}=-6.40848 \\
& \overline{\mathcal{Y}}_{e q} \in(121.62,285.89) \\
& \overline{\mathcal{U}}=(124.96,325.04) \\
& X_{n} \in(0.00,300.00)
\end{aligned}
$$

Figure 8 shows the evolution of the plant controlled by the $M P C \# 2$. In order to introduce more disturbances the measure of the pyrometer was affected. It can be seen how the evolution remains inside the predicted limits with the exception where the estimated error becomes bigger than 5 , this is because the junction of the end of the period were the measure of the pyrometer was disturbed and the effect of a cloud.

Anyway the error can be 13.7275 (the size of $\mathcal{Z}$ ) that

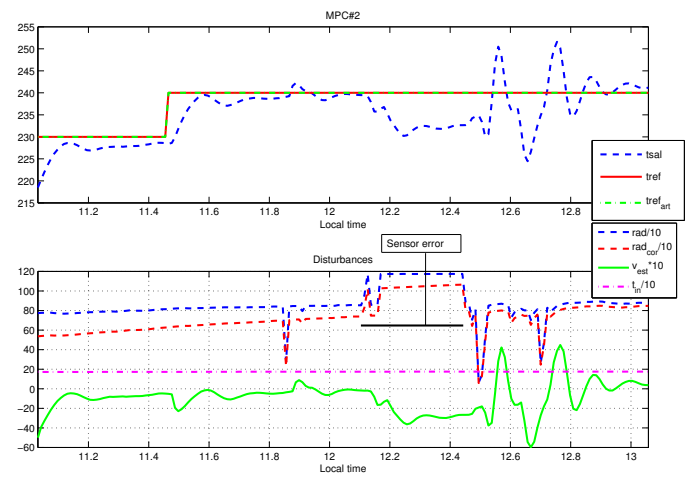

Fig. 8. $M P C \# 2$ with an error in the pyrometer.

can be admissible or not, in the following section a pro- cedure to avoid this error if the disturbance is constant is introduced.

\section{CANCELLATION OF THE TRACKING ERROR}

Due to this controller allows to change the setpoint, this one can be modified, taking into account the value of the disturbance (provided by an estimator), to remove the effect of the disturbance on the output (Limon et al. [2007]). The modified setpoint has the expression:

$$
\begin{aligned}
& \hat{s}(k)=s-[C+D K]\left(I_{n}-(A+B K)\right)^{-1} \hat{w}(k) \\
& \hat{s}(k)=s-H_{s} \hat{w}(k)
\end{aligned}
$$

where $\hat{s}$ is the setpoint that should be provided to make the real system tends to desired one $s, \hat{w}$ is an estimation of the disturbance $w$.

In order to provide a measure of the error an estimator is needed. In this paper the disturbance estimator proposed in (Normey-Rico and Camacho [2007]) is going to be used (DTC discrete disturbance observer):

$$
\begin{aligned}
& w^{+}=b \cdot w-A \cdot \eta+\frac{(1-b) \cdot\left(y^{+}-y_{\text {model }}^{+}\right)}{C} \\
& \eta=(1-b) \cdot \frac{\left(y-y_{\text {model }}\right)}{C} \\
& x_{\text {model }}^{+}=A x_{\text {model }}+B u \quad y_{\text {model }}=C x_{\text {model }}+D u
\end{aligned}
$$

Where $b$ is the constant of a first order filter with the appropriate value.

Figure 9 shows the general scheme.

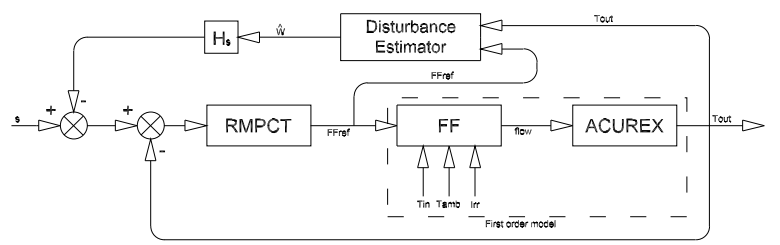

Fig. 9. Tracking error cancellation loop.

The controller that is going to be used is the $M P C \# 1$, the $M P C \# 2$ was so aggressive and working with the DTC generates overshooting.

\section{$6.1 T_{\text {in }}$ disturbance}

Figure 10 shows the plant controlled by the $M P C \# 1$ using the method proposed in section (6). It can be seen how is following the reference in a day with a very good conditions. In order to see the disturbance rejection the temperature in the input was disturbed.

It can be seen how the feedforward overreacts under this disturbance and how the RMPCT is attenuating this effect.

\subsection{Pyrometer sensor disturbance}

Figure 11 shows the response of the controlled plant when an error in the a sensor happens. Using the proposed method, the effect of the disturbance is rejected, without using it, the effect is attenuated but not removed (see figure 8). 


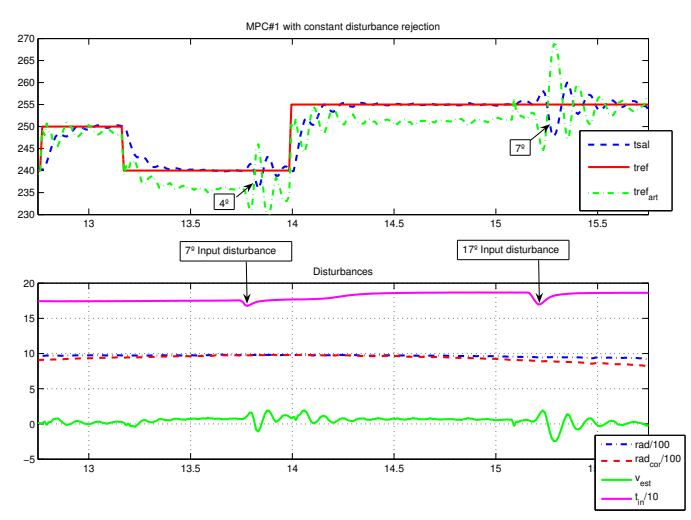

Fig. 10. $M P C \# 1$ with constant disturbance rejection.
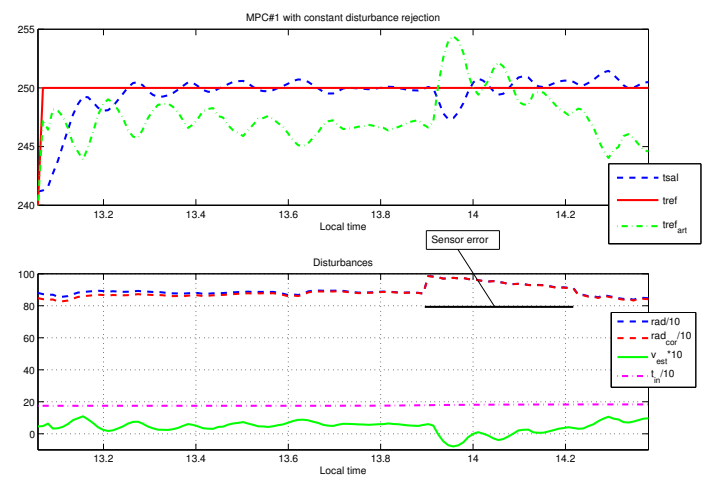

Fig. 11. $M P C \# 1$ with constant disturbance rejection.

\subsection{Radiation disturbance}

Figure 12 shows the response of the controlled plant when there is a strong disturbance in the radiation. Before the
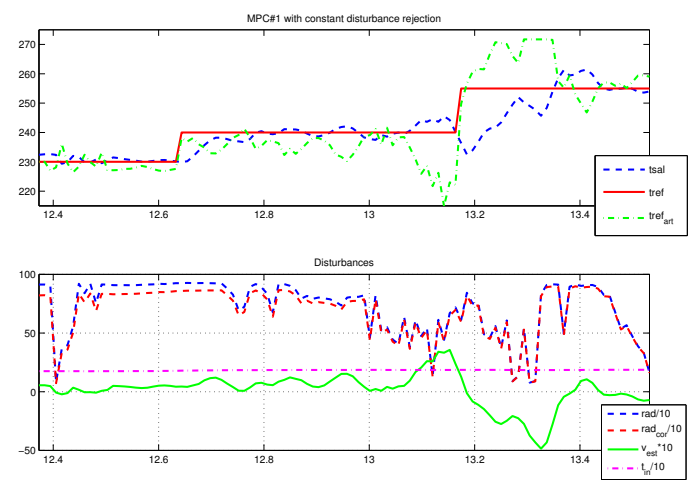

Fig. 12. $M P C \# 1$ with constant disturbance rejection.

second reference change, it looks like the trajectory goes out of the tube, but as it was commented before, in the transient this can happen due to this tube is not centered in the nominal trajectory, is centered in the reference trajectory.

\section{CONCLUSIONS}

A robust controller for processes with unknown but bounded errors has been presented. The proposed control scheme uses a robust identification method and a finite horizon robust model predictive controller for tracking. The robust control algorithm has been tested under simulation and in a distributed solar collector field, which is a process characterized by large perturbations and changes in dynamics caused by clouds and operating conditions. The computational requirements of this type of controller are quite small due to only a QP have to be solved online. The constant disturbance rejection scheme presents the advantage of been able to reject the effect of constant disturbances, but it can inherit problems of the estimator.

\section{REFERENCES}

I. Alvarado. Model Predictive Control for Tracking Constrained Linear Systems. $\mathrm{PhD}$ thesis, Universidad de Sevilla, 2007.

M. Berenguel, E. F. Camacho, and F. R. Rubio. Simulation software pacage of the acurex field. In Internal Report, volume GAR/93-01, 1993.

F Blanchini. Set invariance in control. Automatica, 35: 1747-1767, 1999.

E. F. Camacho and C. Bordons. Model Predictive Control. Springer-Verlag, 2nd edition, 2004.

Camacho E. F., M. Berenguel, and C. Bordons. Adaptative generalized predictive control of a distributed collector field. IEEE Transactions on Automatic Control, CST-2: 462-467, 1994a.

Camacho E. F., M. Berenguel, and F. R. Rubio. Application of a gain scheduling generalized predictive controller to a solar power plant. Control Eng. Pract., 2: 227-238, 1994b.

I. Kolmanovsky and E. G. Gilbert. Theory and conmputation of disturbance invariant sets for discrete-time linear systems. Mathematical Problems in Engineering: Theory, Methods and Applications, 4:317-367, 1998.

D. Limon, T. Alamo, and E.F. Camacho. Enlarging the domain of attraction of MPC controllers. Automatica, 41:629-645, 2005a.

D. Limon, I. Alvarado, T. Alamo, and E.F. Camacho. Robust tube based mpc for tracking of piece-wise constant references for constrained linear systems with aditive disturbances. In Proceedings of the CDC, 2007.

D. Limon, I. Alvarado, T. lamo, and E.F. Camacho. $\mathrm{Mpc}$ for tracking of piece-wise constant references for constrained linear systems. In Proceedings of the IFAC World Congress, 2005b.

D. E. Luenberger. Programacin lineal y no lineal. AddisonWesley Iberoamericana, 1989.

D. Q. Mayne, M. Seron, and S. V. Rakovic. Robust model predictive control of constrained linear systems with bounded disturbances. Automatica, 41:219224, 2005.

Julio E. Normey-Rico and Eduardo F. Camacho. Control of Dead-time Processes. Springer Verlag, 2007.

S.V. Rakovic. Minkowski algebra and banach contraction principle in set invariance for linear discrete time systems. In Proceedings of the CDC, 2007.

S.V. Rakovic, E.C. Kerrigan, K.I. Kouramas, and D.Q. Mayne. Invariant approximations of the minimal robustly positively invariant sets. IEEE Transactions on Automatic Control, 50:406-410, 2005. 